\title{
Coherent Summation of Emission from Relativistic Cherenkov Sources as a Way of Production of Extremely High-Intensity Microwave Pulses
}

\author{
Naum S. Ginzburg, Adrian W. Cross, Anton A. Golovanov, Alan D. R. Phelps, Member, IEEE, \\ Ilya V. Romanchenko, Vladislav V. Rostov, Konstantin A. Sharypov, Valery G. Shpak, \\ Sergey A. Shunailov, Marat R. Ul'masculov, Michael I. Yalandin, Member, IEEE, and Irina V. Zotova
}

\begin{abstract}
For relativistic Cherenkov devices we investigate the process of high-power microwave (HPM) pulse generation with its phase correlating to the sharp edge of an e-beam current pulse. Our theoretical consideration is referred to quasistationary and superradiative (SR) generation regimes when spontaneous emission of the e-beam edge serves as the seed for the development of further coherent oscillations. Phase correlation of the excited microwave pulses with the characteristics of the current pulse front and/or an initial external electromagnetic pulse provided additional confirmation of particle-in-cell (PIC) simulations. Pulse-to-pulse stability of the radiation phase within several percent of the oscillation period makes it possible to arrange multi-channel schemes producing mutually coherent microwave pulses. In the experiments that have been carried out, the cathodes of independent generators were powered by identical accelerating pulses from strictly synchronized voltage modulators, or by splitting the pulse from a single powerful modulator. For the 2-ns regime with the power of each Ka-band backward wave oscillator (BWO) about $100 \mathrm{MW}$, we demonstrate quadratic growth of the power density in the interference maximum of the directional diagram. In a short pulse SR regime, with the peak power of $600 \mathrm{MW}$ in a single channel, for a four-channel 2-D array we attained a sixteen-fold radiation intensity gain.
\end{abstract}

Index Terms - superradiance of electron bunches, current edge, microwaves, radiation phase, coherent summation.

\section{INTRODUCTION}

$\mathrm{P}$ ROSPECTIVE scientific and technological applications stimulate interest in the generation of ultra-high power

Manuscript received August 31, 2015. The work was supported by IEP UB RAS Project No. 0389-2014-0005 and, in part, by RFBR Grants Nos. 14-0801180 and 16-02-00029.

N. S. Ginzburg, A. A. Golovanov and I. V. Zotova are with the Institute of Applied Physics RAS, N. Novgorod 603950, Russia (e-mail: ginzburg@appl.sci-nnov.ru).

A. W. Cross and A. D. R. Phelps are with the Department of Physics, SUPA, University of Strathclyde, Glasgow G4 0NG, U.K. (e-mail: a.d.r.phelps@strath.ac.uk).

I. V. Romanchenko and V. V. Rostov are with the Institute of High Curren Electronics, SD RAS, Tomsk, 634055, Russia (e-mail: rostov@lfe.hcei.tsc.ru).

K. A. Sharypov, V. G. Shpak, S. A. Shunailov, M. R. Ul'masculov, and M. I. Yalandin are with the Institute of Electrophysics, UD RAS, Ekaterinburg, 620016, Russia (e-mail: yalandin@iep.uran.ru). coherent radiation. Approaches that can be suggested to achieve this goal include the generation of radiation by a single source with an oversized electrodynamic system. In this case special methods (for example, 2D distributed feedback [1], [2]) are required to produce spatially coherent radiation. Other methods are based on time-amplitude HPM power compression schemes [3] and on the synchronization of a large number of moderate-power sources using a master oscillator [4]-[6].

At the same time for short-pulse sources, in particular, for HPM oscillators based on Cherenkov radiation from relativistic electron beams moving in a slow wave structure (SWS) [7], [8], there is an alternative opportunity associated with correlating the phase of a radiated pulse to the sharp edge of a current pulse. Actually, spontaneous emission of the bunch edge serves as the seed for stimulated processes that include electron self-bunching and subsequent radiation of a high-power electromagnetic (EM) pulse. If identical current pulses are sent simultaneously to several oscillators' channels, identical in-phase microwave pulses are generated and the coherent summation of their amplitudes is possible.

In fact, the methods of phase synchronization have been experimentally tested for two different types of operation regime. The first way is a conventional regime of a relativistic BWO with quasi-stationary generation [9]. In such a regime the parameters of generation (frequency and electron efficiency) are the same as in the steady-state regimes. Nevertheless, the phase of the generated signals is strongly correlated with the sharp edge of a current pulse. The second operating mode represents a regime of superradiance when the e-beam current pulse length is comparable with the cooperative length (see details in [7-8]), and such an electron bunch emits a single radiation pulse with the peak power exceeding the power in a steady-state regime. It is obvious that for both mentioned operating regimes the phase coupling to the sharp edge of a current pulse permits one to control the phase by a precise time shift in the beam current front.

It should be noted that the physical model describing the transformation of spontaneous Cherenkov radiation (i.e. the radiation from unperturbed moving particles without the reverse effect of the field [10]) to stimulated radiation have been suggested in Ref. [11]. Development of such an 
analytical model (Section II), related PIC-simulations (Section III) and presentation of experimental results (Section IV) are the aims of this paper. In experiments carried out, for the first time a two-dimensional array consisting of four synchronized Cherenkov SR generators has been realized, which allows extremely high EM fields in the millimeter band to be obtained.

\section{Theoretical Model. Averaged APPROACH.}

\section{A. Basic Equations}

The basic physics of the generation initiated by the radiation from the sharp edge of a current pulse can be understood from the fundamental model shown in Fig. 1 (a). We will assume that an extended electron bunch with length $l_{b}$ moves with the longitudinal velocity $v_{0}=\beta_{0} c$ along a strong magnetic field in a planar waveguide with periodically corrugated walls: $b(z)=b_{1} \sin (\bar{h} z)$, where $b_{1}$ is the corrugation amplitude, $\bar{h}=2 \pi / d, d$ is the corrugation period. Under the assumption of small corrugation amplitude $b_{1}<<d$ the structure of the radiated field is close to a TEM mode of the regular waveguide. However, since the electric field should be perpendicular to the metal walls of the slightly curved waveguide a small longitudinal component of the electric field appears:

$$
E_{z}=-\bar{h} b_{1} \cos (\bar{h} z) E_{x} .
$$

Excitation of the TEM mode by an electron bunch can be described by the following equation [12]:

$$
\frac{\partial^{2} E_{x}}{\partial z^{2}}-\frac{1}{c^{2}} \frac{\partial^{2} E_{x}}{\partial t^{2}}=-\frac{4 \pi}{c^{2}} \frac{\bar{h} b_{1}}{b} \cos (\bar{h} z) \frac{\partial}{\partial t} J,
$$

where $b$ is the gap between the waveguide walls and $J$ is the electron current.

We will represent the electron bunch as a set of macroparticles with a small length $\Delta l$ and different initial positions over the longitudinal axis. The current of the macroparticle with the initial coordinate $z_{0}$ can be written as $J=\sigma \Delta l \delta\left(t-\left(z-z_{0}\right) / v_{0}\right)$, where $\sigma=\int_{-b / 2}^{b / 2} \rho d x, \rho$ is the charge density, $\delta(x)$ is the Dirac delta function. According to the Eq. (2) the field excited in the waveguide by the current $J$ is given by the relations:

$$
E_{x}^{ \pm}=\frac{2 \pi k_{ \pm} b_{1} \sigma \Delta l}{b} \cos \left[\omega_{ \pm}\left(t+\frac{z_{0}}{v_{0}} \mp \frac{z}{c}\right)\right] .
$$

where $\omega_{ \pm}=\bar{h} v_{0} /\left(1 \mp \beta_{0}\right), k_{ \pm}=\omega_{ \pm} / c$. The upper sign in Eq. (3) corresponds to radiation in the forward direction $\left(z>z_{0}+v_{0} t\right)$, and the lower sign - to radiation in the backward direction $\left(z<z_{0}+v_{0} t\right)$. Thus a single particle moving through a corrugated waveguide radiates a highfrequency (HF) component in the direction of the electron movement and a low-frequency (LF) component in the opposite direction. The field radiated by the whole bunch is obtained by summation of the fields emitted by the individual macroparticles. In the approximation of the unperturbed motion of the electrons this summation is reduced to the interference of the radiation from the front and trailing edges of the electron bunch. This emission should be interpreted as spontaneous with the total radiation power in the operating TEM mode defined by the formula:

$$
P^{ \pm}=\frac{\pi b_{1}^{2} \sigma^{2} v_{0}^{2}}{c b} \sin ^{2}\left(\frac{\omega_{ \pm}}{2 v_{0}} l_{b}\right) \text {. }
$$

(a)

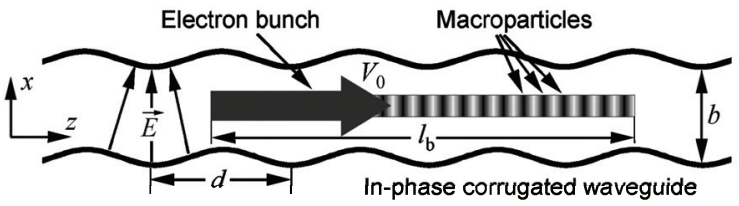

(b)

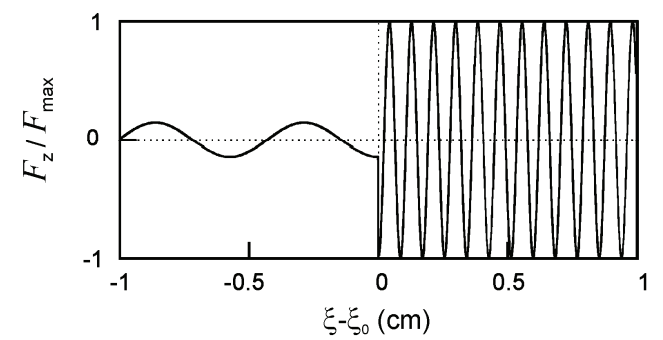

Fig. 1. (a) Model used for analysis of initiation of microwave generation by spontaneous emission from the leading edge of an electron beam moving in a periodically corrugated waveguide. (b) Longitudinal profile of an average force, with which the selected macroparticle acts on electrons in other macroparticles.

Significantly higher radiation power can be achieved if the particles' self-bunching is taken into account. This process is caused by the mutual influence of the electrons and, in fact, develops from the spontaneous emission mentioned above. For further analysis it is convenient to introduce new independent variables $\xi=z-v_{0} t, \tau=t$ in which the longitudinal coordinate is moving with the electron unperturbed velocity. In these coordinates the field radiated by the macroparticle with the initial coordinate $\xi_{0}=z_{0}$ is written in the following form:

$$
E_{x}^{ \pm}=\frac{2 \pi k_{ \pm} b_{1} \sigma \Delta l}{b} \cos \left[\Omega \tau \mp k_{ \pm} \xi+\frac{k_{ \pm} \xi_{0}}{\beta_{0}}\right],
$$

where $\Omega=\bar{h} v_{0}$. Using the new variables and substituting Eq. (5) in Eq. (1) we derive the average longitudinal force with which the selected macroparticle acts on electrons in other macroparticles:

$$
\tilde{F}_{z}^{ \pm}\left(\xi-\xi_{0}\right)=-\frac{\pi e k_{ \pm} \bar{h} b_{1}^{2}|\sigma| \Delta l}{b} \cos \left(\frac{k_{ \pm}\left(\xi-\xi_{0}\right)}{\beta_{0}}\right),
$$

where the upper sign "+" corresponds to the region $\xi>\xi_{0}$, and the lower sign "-" to the region $\xi<\xi_{0}$. 
Thus, in the frame of our model the electron bunch is described as gas of macroparticles interacting via the longitudinal force (6) with a profile shown in Fig. 1 (b). It is important to note that this force has an alternating sign, which leads to the development of self-bunching of the electrons. This process can be described by the averaged equations of the particles' motion in the form:

$$
\begin{aligned}
& \frac{d p_{i}(\tau)}{d \tau}=\sum_{j \neq i} \tilde{F}_{z}\left[\xi_{i}(\tau)-\xi_{j}\left(\tau-\tilde{\tau}_{j i}\right)\right], \\
& \frac{d \xi_{i}(\tau)}{d \tau}=\frac{p_{i}(\tau)}{m \gamma_{i}(\tau)}-v_{0},
\end{aligned}
$$

where

$$
\tau_{j i}=\left|\xi_{i}(\tau)-\xi_{j}(\tau)\right| /\left(c \pm v_{0}\right)
$$

is the propagation delay of the $j$-th electron radiation at the position of the $i$-th electron. The total electromagnetic field, radiated by the whole bunch, can be found as a sum of the fields (3), emitted by a single electron.

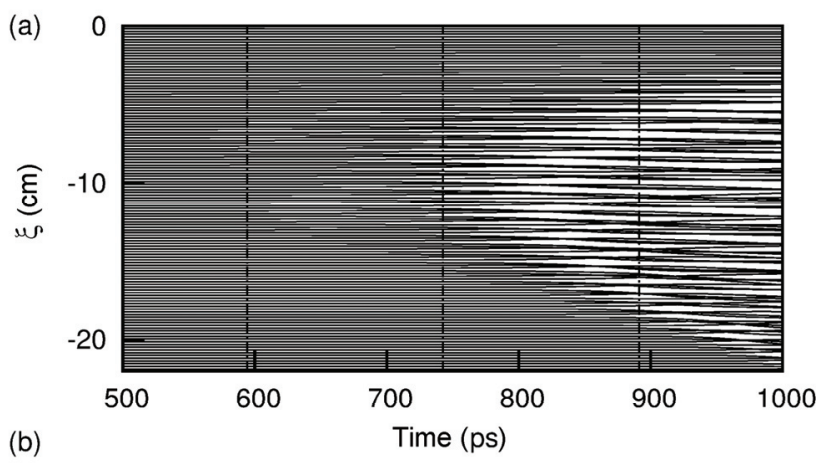

(b)

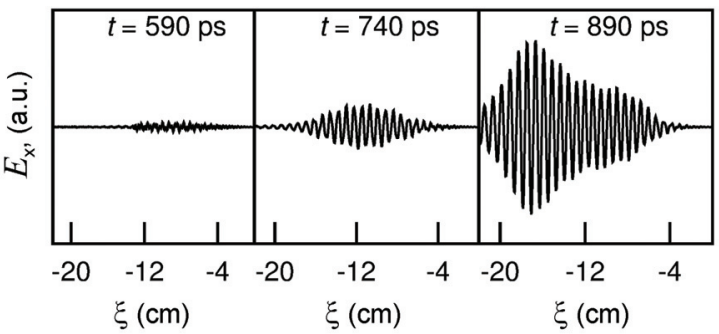

Fig. 2. (a) Electrons' self-bunching and (b) radiated field longitudinal profile at different moments in time.

\section{B. Initiation of Superradiance Pulse generation}

Simulations of superradiance initiated by the radiation of an electron bunch edge were performed for the electron energy of $250 \mathrm{keV}$, the line current density of $850 \mathrm{~A} / \mathrm{cm}$, the gap between the waveguide plates of $0.2 \mathrm{~cm}$, the corrugation period of $0.33 \mathrm{~cm}$, and the corrugation amplitude of $0.013 \mathrm{~cm}$. Under these parameters, the operating frequency was approximately $38 \mathrm{GHz}$. The current pulse duration was considered to be equal to $1 \mathrm{~ns}$. The duration of the current pulse edges was approximately 300 ps. The self-bunching of electrons arising under the influence of the averaged force (6) is shown in Fig. 2 (a). In the simulations it is observed that the backward wave radiation with a low frequency has the decisive influence on the particles' motion. Correspondingly, the electrons are bunched in such a way that this component of radiation is amplified. The instantaneous distribution of the radiated field is shown in Fig. 2 (b). At the beginning (at $t=590 \mathrm{ps}$ ) it is spontaneous and caused by the radiation of the bunch front edge. Then the particles' self-bunching development leads to the amplification of the LF component and the generation of a SR pulse emitted in the $-\mathrm{z}$ direction (at $t=740 \mathrm{ps}$ and $t=890 \mathrm{ps}$ ). It is seen that the peak power of this LF component [Fig. 2 (b), at $t=890 \mathrm{ps]}$ significantly exceeds the power of the spontaneous radiation 'Fig. 2 (b), at $t=590 \mathrm{ps}]$, as given by Eq. (4). At the same time the amplitude of the HF component radiated in the $+z$ direction remains practically constant at the level determined by the formula (4).

\section{Initiation of Long-Pulse (Quasi-Stationary) Generation}

It is also possible to apply our simple model for the description of quasi-stationary emission initiated by the front edge of an electron bunch (beam) that is longer than the interaction space. In this case the electrons leaving the SWS interrupt their interaction with the other electrons and so do not participate further in the radiation process. Therefore such electrons can be eliminated from consideration. Depending on the electron bunch current and the length of the interaction space, it is possible to obtain three generation regimes: stationary, periodic self-modulation and stochastic selfmodulation. The backward-radiated fields obtained in simulations for these three regimes of BWO operation are shown in Fig. 3. The parameters of the SWS and the electron energy were taken the same as in subsection B.
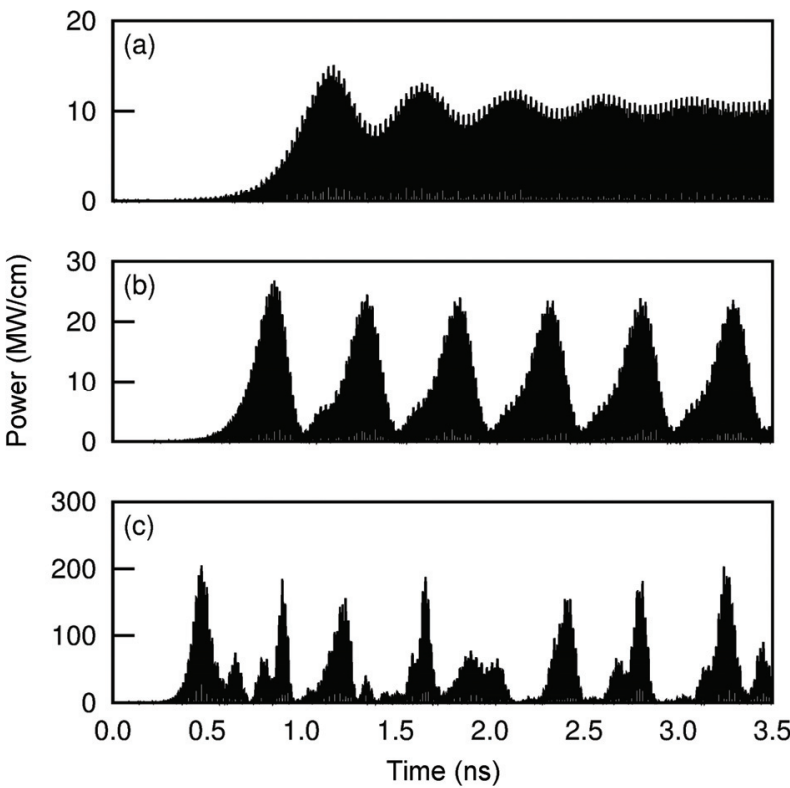

Fig. 3. Backward-radiated field of (a) stationary, (b) periodic self-modulation and (c) stochastic self-modulation regimes. The electron bunch line current density and the length of the interaction space are: (a) $450 \mathrm{~A} / \mathrm{cm}$ and $4 \mathrm{~cm}$; (b) $450 \mathrm{~A} / \mathrm{cm}$ and $4.8 \mathrm{~cm}$; (c) $1400 \mathrm{~A} / \mathrm{cm}$ and $6 \mathrm{~cm}$, respectively. 


\section{PIC SiMULATIONS}

In addition to the above analytical consideration using an average approach (Sect. II), we performed direct particle-incell simulations (code KARAT [13]) which give more evidence regarding the correlation of the phase-temporal structure of the radiated microwave pulses with the characteristics of the beam current front. Moreover, below we present simulation results related to additional mechanisms of phase control based on the injection of an external short EM pulse.

\section{A. SR Pulse Initiation by Leading Edge of Electron Beam}

The model of the Ka-band SR generator used in the simulations (geometry of e-beam injector, cylindrical corrugated SWS and topology of guiding magnetic field) is identical with that exploited in the experiments [see Fig. 4].

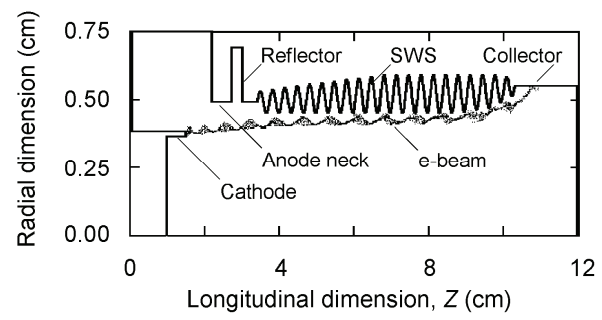

Fig. 4. Geometry of a non-stationary (superradiance) Ka-band BWO model used in PIC-simulations.
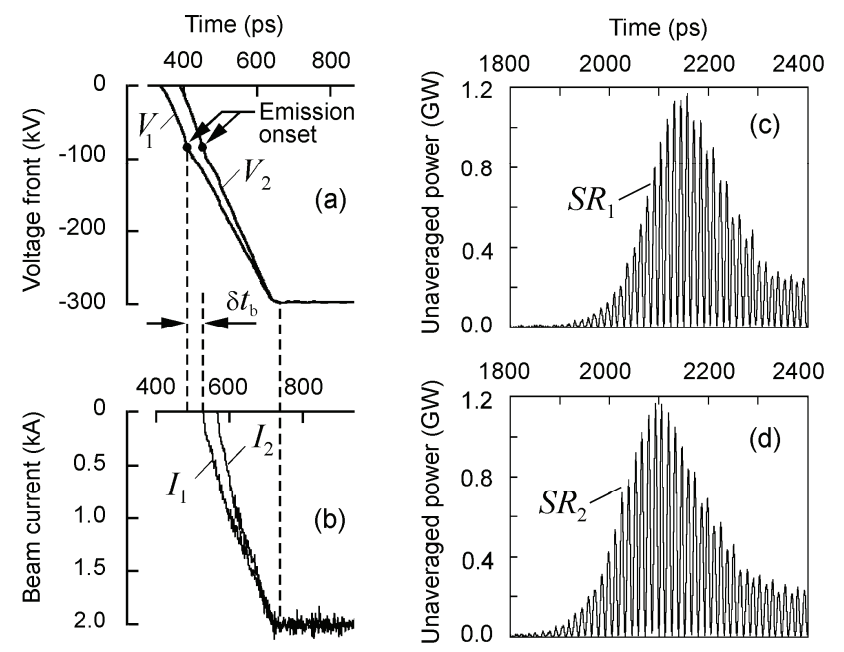

Fig. 5. (a) Leading edge of the voltage pulses with different rise time applied to the cathode. (b) Corresponding beam current fronts at the SWS inlet $(\mathrm{z}=3.5 \mathrm{~cm})$ as it responds to the voltage sharpening (a). (c,d) Instantaneous power of SR pulses for voltage and current fronts $\left(V_{1} ; I_{1}\right)$ and $\left(V_{2} ; I_{2}\right)$, respectively.

In the simulations, an infinite cathode emissivity approximation was applied. However, the beam emission onset at the voltage leading edge $V_{1}$ which rises linearly up to $-300 \mathrm{kV}$ for $300 \mathrm{ps}$ [Fig. 5 (a)] accounted for the delay of the explosive electron emission development at the graphite cathode [14]. It is important that the time of the electrons' acceleration in the "cathode-anode" gap is comparable to the rise time of the voltage pulse. As a result, kinematic effects [15] provide sharpening of the current $I_{1}$ front at the inlet of the SWS [ $z=3.5 \mathrm{~cm}$ in Fig. 4] by $\delta t_{b} \approx 50$ ps [Fig. 5 (b)] as compared to the voltage $V_{1}$ leading edge.

Additional sharpening of the voltage front (compare $V_{2}$ and $V_{1}$ ) leads to the further shortening of the e-beam current edge $\left(I_{2}\right)$. This is accompanied with the rise of $d I / d t$ at the SWS input and, hence, increases the intensity of the high-frequency spectral components of the wideband initial spontaneous EM signal generated by this current front. A certain spectral component of the signal satisfies Cherenkov beam-wave synchronism condition and stimulates development of SR emission [16], [17]. This suggested physical mechanism explains the reason why the superradiative pulse $S R_{2}$ in Fig. 5 (d) leads to the nearly similar pulse $S R_{1}$ in Fig. 5 (c) while, on the contrary, the beam current edge of $I_{2}$ is delayed to some extent with respect to the current edge $I_{1}$.

\section{B. Quasi-Stationary BWO Generation Initiated by Beam Current Front}

A distinctive feature of the BWO configuration providing quasi-stationary generation was a 40-percent reduced SWS length as compared to that shown in Fig. 4. This leads to an almost five-fold rise in the starting current required for generation [see Refs. [9] and [18], and citations therein] when the parameters of the electron injector and the characteristics of the electron beam were nearly identical to those described above.

In the PIC simulations, phase correlation of the microwave pulse generated in the BWO is an obvious effect when the beam current edge retains its shape. Naturally, however, development of the generation is delayed to some extent with respect to the driving electron pulse. Figure 6 (a) demonstrates the build-up of quasi-stationary generation in the BWO with the beam power of $\sim 450 \mathrm{MW}$ and the electron efficiency exceeding 35\% [19]. This case corresponds to the results of the analytical model shown in Fig. 3 (a). However, even minor variations in the accelerating voltage front shape may change the onset time (and the phase) of the microwave pulse formation. For the purposes of illustration, we simulate the influence of a preceding voltage pre-pulse [dashed circle in Fig. 6 (c)] which is typical for power modulator schemes with output sharpening spark gaps. Due to a fast voltage rise at the front of the main accelerating pulse, the electrons emitted during the pre-pulse form an advanced current burst at the end of the drift space [compare Figs. $6(\mathrm{~d})$ and $6(\mathrm{e})$ ]. This is sufficient to shift the front of the microwave pulse, that had already formed by almost 100 ps [compare Figs. 6 (f) and $6(\mathrm{~g})]$. 

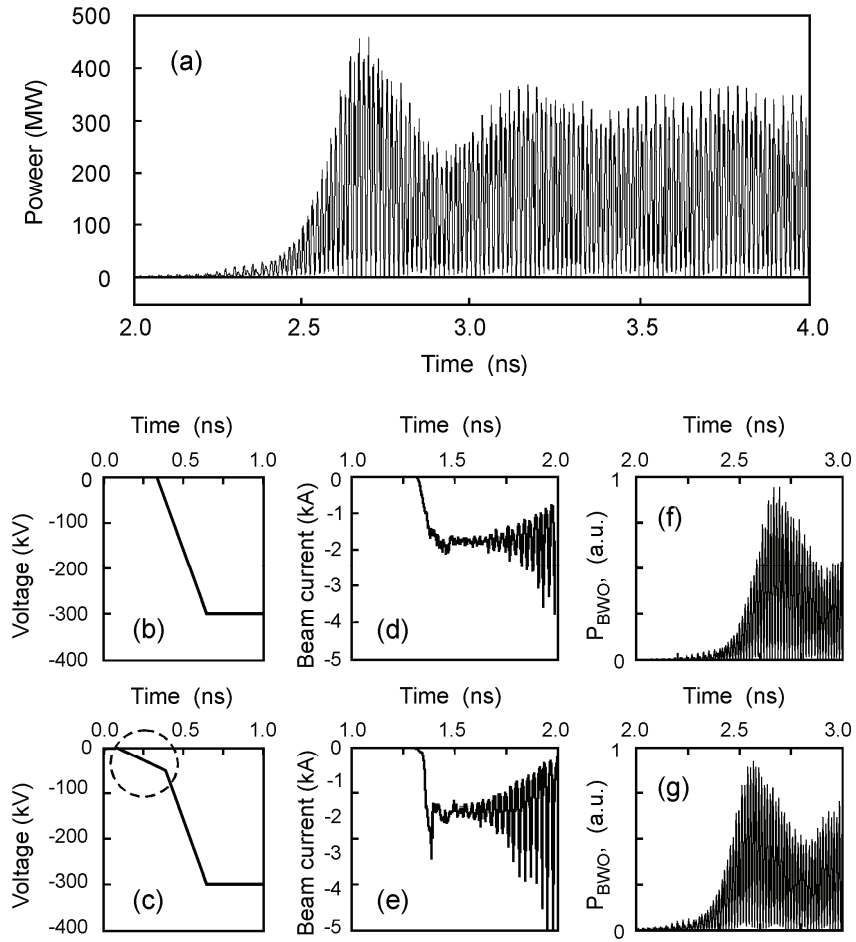

Fig. 6. (a) Instantaneous power of quasi-stationary Ka-band BWO. $(b, c)$ Leading edges of the voltage pulses with different shape. $(d$, e) Current fronts at the SWS output and (f, g) microwave pulse fronts corresponding to (b) and (c), respectively.

\section{Initiation of SR Pulse Emission by an External EM Pulse}

Figure 7 presents numerical simulations of initiation of SR pulse emission stimulated by an external short EM pulse. It contains only five oscillation periods at half magnitude level. In fact, the rise time of the bell-shape pulse envelope was ten times shorter as compared to the beam current leading edge (this front was specially extended to $\sim 1 \mathrm{~ns}$ ). Besides, the width of the external pulse was much less than the time of its propagation along the SWS and approximately 2 times shorter than the duration/front of the excited superradiative pulse [Fig. 7 (b)].

The following conditions should be satisfied for the effective action of a short seed microwave pulse. The power of this pulse should exceed a level of signal from the current edge (i). The "meeting" of a seed pulse peak and the current front should be provided near the SWS input (ii), and at the moment of time when the electron energy at the current front attains the band of beam-wave Cherenkov synchronism (iii). Under such conditions for the power ratio of $\sim 1.5 \times 10^{-4}$ we clearly observed correlation of phases between the seed wave pulse and the excited $S R_{1}$ pulse. Figures 7 (c) and 7 (d) demonstrate identical phase shift from 0 to $\pi$. Confirmations for intermediate shifts were also obtained.

In agreement with results [20], [21], where the influence of an external signal on operation of a long pulse relativistic BWO was studied, we also observed that in the presence of a seed pulse, the pulse $S R_{1}$ formation occurs somewhat earlier than in the case of the free-running regime [see delayed dashed envelope of the pulse $S R_{2}$ in Fig. 7 (b)].

The use of an ultra-short seed EM pulse to control the phase of the SR source means that several such radiators could be phase-synchronized from a single HPM source. As the peak power of a seed signal is only tens of kilowatts then many identical signals could be formed by splitting a single pulse from a master SR generator. This generator could be very similar to these under control. Thus, multi-channel, phaseadjustable arrays of SR generators could be created and utilize effectively the power of multi-gigawatt and even subterawattrange voltage modulators which usually form accelerating pulses with the rise time exceeding $1 \mathrm{~ns}$. Another advantage of an external signal's phase control is the decreased stability requirements of the voltage and current fronts.

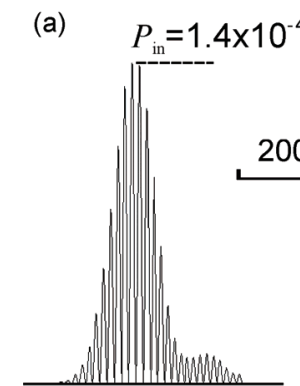

(b)
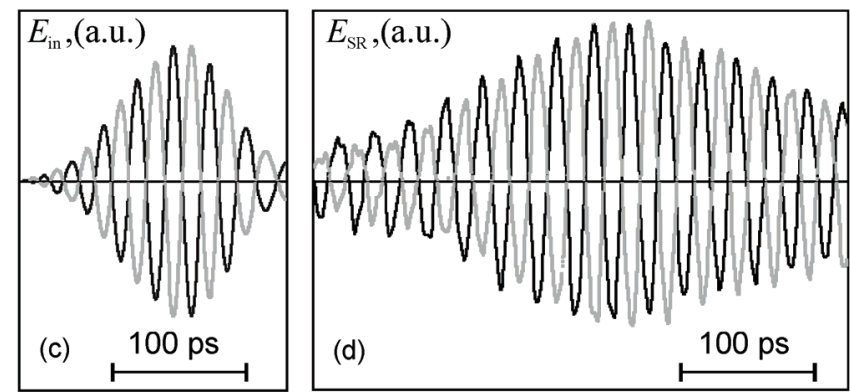

Fig. 7. (a) Non-averaged power $P_{\text {in }}$ of a seed microwave pulse. (b) Corresponding superradiative pulse $\mathrm{SR}_{1}$ with peak power $P_{\mathrm{SR}}$ and the same pulse $\mathrm{SR}_{2}$ obtained for free-running regime of the oscillator. (c) and (d) Correlated phase shifts ( 0 to $\pi$ ) for electric fields of seed and SR microwave Ka-band pulses, respectively.

\section{EXPERIMENTS WITH IN-PHASE BWOS}

\section{A. Experimental Layout}

Both quasi-stationary and superradiative Ka-band BWOs were tested in multi-channel configuration. In the experiments, the closely identical nanosecond accelerating pulses that were applied to the explosive emission graphite cathodes were produced by two methods. In the first studies [22], two RADAN-303 high voltage drivers [23] based on a double pulsed forming lines were charged in parallel and then switched by a common, controllable gas spark gap to independent BWOs. The second device was built on the basis 
of a more powerful all-solid-state modulator with inductive energy storage and a semiconductor opening switch (SOS) [24]. It was capable of driving up to four parallel Ka-band BWOs by a split voltage pulse. In both cases, the voltage pulse formed in each channel was additionally sharpened using a coaxial gyromagnetic nonlinear transmission line (NLTL) [25]. Steepness of the voltage rise time and its delay were controlled precisely [Fig. 8] with the change in saturation of the ferrite rings assemblies placed in the oil-filled insulation gap of the lines. To achieve this, an axial magnetic bias was independently varied by readjustment of the current in $\mathrm{dc}$ solenoids wound around the NLTLs.

In accordance with the consideration given in Section II and the PIC simulations presented in Figs. 3 and 6, for coherent summation of microwave pulses from independent sources where the radiation phase is correlated with the beam current front, it is necessary to provide the stability of the beam formation within picoseconds. Fortunately, the possibility of such accuracy was practically demonstrated [26] and fully used in the experiments described below.

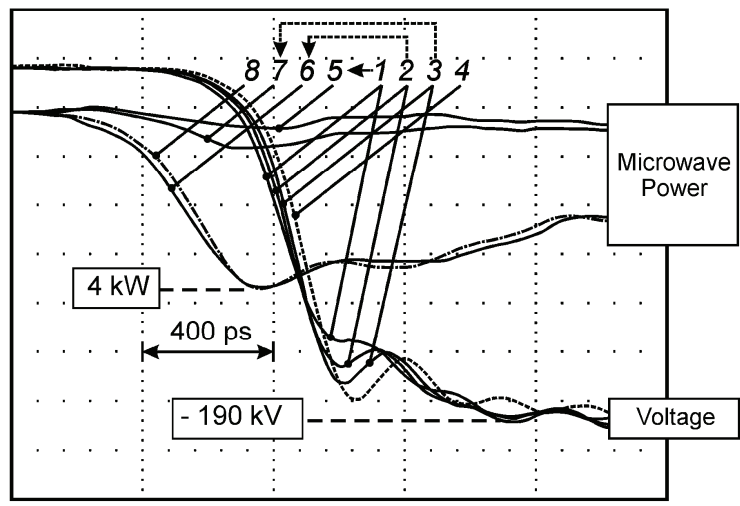

Fig. 8. Variation in the voltage front steepness and delay in the tested channel after its passage through the NLTL (1-3) with adjustment of the solenoid supply current (2.5 A; $3.25 \mathrm{~A}$ and $4.0 \mathrm{~A}$, respectively) as compared with the front in the reference channel (4) where the current of 5 A was unchanged. Waveforms from the microwave detector (5-7) were recorded with $-6 \mathrm{~dB}$ (four-fold) attenuation of the radiation power when two independent BWOs operated in-phase (6) and in counter-phase regime $(5,7)$. Signal (8) corresponds to a single BWO operation in the reference channel without power attenuation. Time reference between the voltage and microwave envelopes is arbitrary. (a)

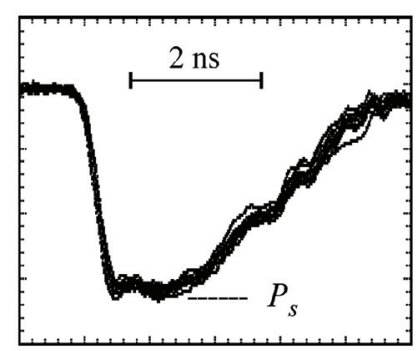

(b)

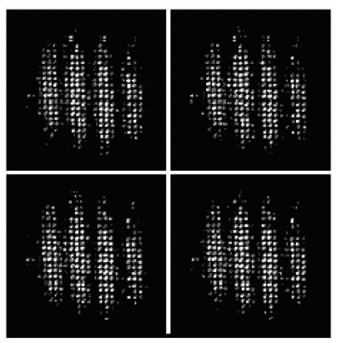

Fig. 9. (a) Accumulation of ten consecutive traces from microwave detector recorded with the signal overlayed from two in-phase BWOs each operated with 100-MW peak power. (b) Reproducible interference luminescence picture on gas-discharge panel in the mode of counter-phase summation of two linearly-polarized Gaussian wave beams.

\section{B. Summation of Nanosecond HPM Pulses from Quasi- Stationary $B W O$}

In the experiment with quasi-stationary BWOs, the transverse structure of the radiation from each oscillator corresponded to the excitation of the $\mathrm{TM}_{01}$ mode of the cylindrical waveguide. In the output section the radiated field was transformed to a Gaussian wave beam. From comparison of powers recorded from a single BWO and from two in-phase oscillators [see nearly identical traces $(8)$ and $(6)$ in Fig. 8], coherent summation of the wave beams in free space leads to a four-fold rise in radiation power density. It is important that such a coherent summation was over a long-term, up to 100 200 periods of the microwave field. These results were reproducible for a set of sequential shots, as presented in Fig. 9. Thus very high stability of electron beams' fronts have been attained, including reproducibility of cathode emission [17] and successful suppression of probable shunting current emission from the stainless-steel cathodes' holders [27].

\section{In-phased Superradiative Oscillators}

In experiments with coherent summation of the emission from two-channels Ka-band superradiative oscillators [28] we demonstrated high stability of the phases ( $\sim 2 \%$ standard deviation). The radiation power density with a single oscillator of power $700 \mathrm{MW}$ was equivalent to $\sim\left(700 \mathrm{MW} \times 2^{2}\right) \approx 3 \mathrm{GW}$ for the two phased synchronised oscillators. It was also noted that the leading SR peak was very reproducible, with the power summation lose coherence for the tail fraction of the microwave signals [see Fig. 10] following the same general trend as given from analytical consideration for BWO's with chaotic generation development [Fig. 3 (c)].

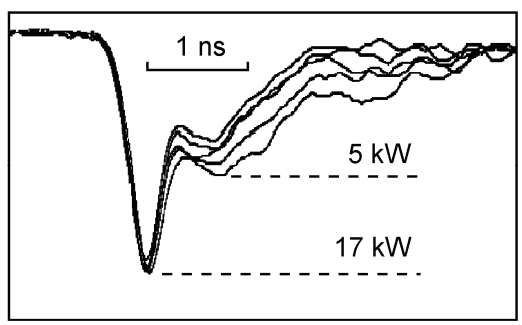

Fig. 10. Signals of non-linear microwave probe demonstrating the loss of coherency in summation of two SR pulses due to generation phase chaoticization following the superradiation peak formation.

Figure 11 demonstrates accelerating voltage pulse and ebeam current (the last was measured by collector probe [15] at the SWS inlet) both practically identical for each electron injector of the four-channel SR BWO. As it was valid earlier in the experiment [28], the fast portion in the rise time of current pulse [rectangle in Fig. $11(\mathrm{~b})$ ] exhibits $d I / d t \geq 5 \mathrm{kA} / \mathrm{ns}$. This parameter is accompanied with electrons' energies [top amplitude inside rectangle in Fig. 11 (a)] which definitely satisfied Cherenkov synchronism. This was sufficient for coherent summation of four SR pulses [11], as it is presented in Fig. 12. Here one can compare the microwave detector signal envelope [Fig. 12 (a)] from one of 
the four SR BWOs (as it was measured in the far field zone at the axis of the 2-D antenna array) and the integral signal [Fig. 12 (b)] obtained with summation of four SR pulses and recorded at the same point of space. The last measurement was made with $-12 \mathrm{~dB}$ attenuation, i.e., the microwave power in the receiver was reduced by sixteen times. Equality of the amplitudes of the signals presented in Figs. 12 (a) and 12 (b) demonstrates a quadratic rise of the radiation power flux density in the maximum of the interference pattern [Figs. 12 (c) and 12 (d)]. Reproducibility of the integral signal from pulse to pulse in Fig. 12 (b) proves the stability of the SR phases of all four BWOs and the coherent nature of the summation.

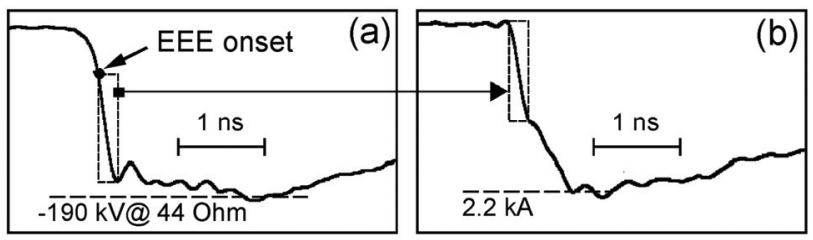

Fig. 11. (a) Sharpened accelerating voltage pulse at 44-Ohm output of NLTL. (b) Electron beam current recorded in the cross-section $\mathrm{z}=3.5 \mathrm{~cm}$ (Fig. 4) by fast collector probe placed instead of SWS.

(a)

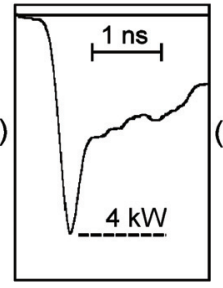

(b)
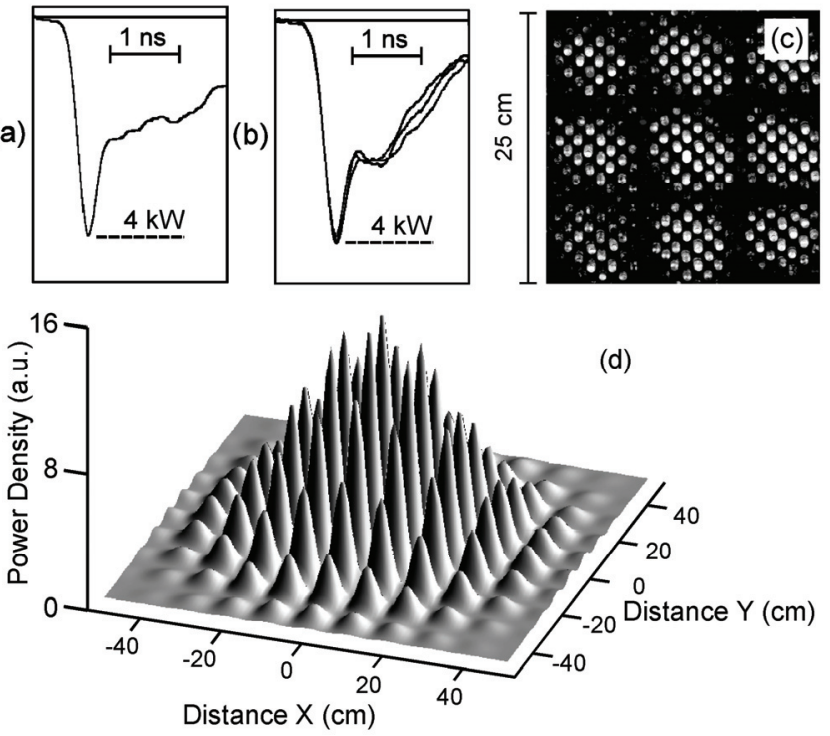

Fig. 12. (a) Microwave pulse recorded by germanium detector from a single SR BWO. (b) Three stored detector's signals of the sum of in-phase SR pulses from 4 generators. (c) and (d) Interference pattern of the radiation recorded by a gas-discharge matrix panel at a distance of $1.5 \mathrm{~m}$ from the array of 4 generators and the power density simulation at the same distance, respectively.

Thus, we attained a power flux density which is equivalent to a single Ka-band microwave oscillator having similaraperture horn antenna and an output power of $\left(600 \mathrm{MW} \times 4^{2}\right)$ $\approx 10 \mathrm{GW}$. This performance was not previously attained if no measures were taken to focus the microwave beam in free space [30]. With that, at a distance of $1 \mathrm{~m}$ from the antennas' array, the maximum power flux density was as high as
$10 \mathrm{MW} / \mathrm{cm}^{2}$, and the strength of the electric field attained there $\sim 140 \mathrm{kV} / \mathrm{cm}$. This is almost five times higher than dc breakdown strength $(30 \mathrm{kV} / \mathrm{cm})$ for atmospheric air at normal conditions. However, no breakdowns were observed in free space within the directional pattern, as for the time interval as short as $\sim 200 \mathrm{ps}$ we obtained earlier [30] the microwave breakdown "threshold" rise above $150 \mathrm{kV} / \mathrm{cm}$. At the same time, there were visually recognizable breakdowns of air near each vacuum window of the horn antennas [Fig 13]. Here, estimates show the E-field maximum value approaches $200 \mathrm{kV} / \mathrm{cm}$. However, the removal of these sparks by using $\mathrm{SF}_{6}$ gas isolation of the windows [31] changed the envelopes of recorded SR pulses [Fig. 12 (a)] very little, if at all. This confirms the incomplete nature of observed subnanosecond $\mathrm{rf}$ breakdowns and gives a chance to increase HPM power density in ambient air to an even higher value.
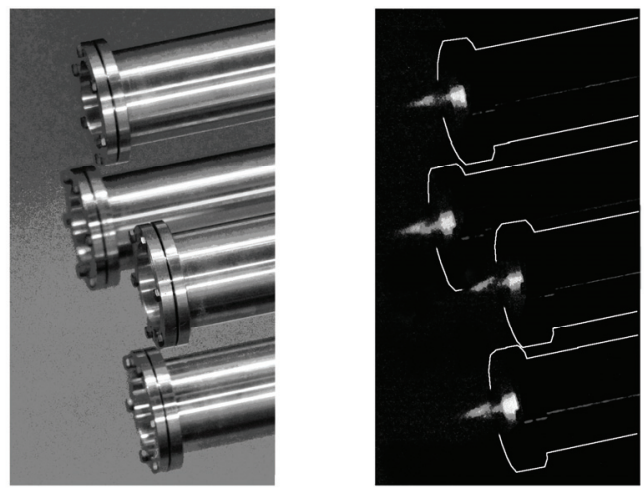

Fig. 13. Array of four horn antennas (left) and incomplete rf breakdowns of air near vacuum vindows at peak SR power of $600 \mathrm{MW}$ in each BWO's channel (right).

\section{CONCLUSION}

Suggested theoretical analysis and direct PIC simulations demonstrate the possibility of initiation of Cherenkov stimulated emission by spontaneous emission of the electron beam leading edge. If identical current pulses are sent simultaneously to several channels, identical in-phase microwave pulses will be generated and the coherent summation of their amplitudes is possible. These methods of phase synchronization have been experimentally tested for two different types of operation regimes. The first one is a conventional regime of relativistic BWO quasistationary generation [9]. The second operation mode represents a regime of superradation when the e-beam current pulse length is comparable with the beam-wave interaction length [7], [29]. For a long pulse $(\sim 2 \mathrm{~ns})$ regime with the power of each Kaband BWO about $100 \mathrm{MW}$, we have demonstrated quadratic growth of the power density in the interference maximum of the directional diagram. In a short pulse superradiative regime with peak power of $600 \mathrm{MW}$ in a single channel, for fourchannel 2-D array we increased the intensity of the microwaves 16 times.

We have grounds to believe that the above innovatory approach opens up new opportunities in the generation of microwave pulses with extremely high power density in multi- 
channel sources with controlled distribution of the highfrequency phase in each of them.

\section{REFERENCES}

[1] N. S. Ginzburg, N. Yu. Peskov, A. S. Sergeev, A. D. R. Phelps, I. V. Konoplev, G. R. M. Robb, A. W. Cross, A. V. Arzhannikov, and S. L. Sinitsky, "Theory and Design of a Free-Electron Maser with TwoDimensional Feedback Driven by a Sheet Electron Beam," Phys. Rev. E, vol. 60, no. 1, pp. 935-945, Jul. 1999.

[2] I. V. Konoplev, P. McGrane, W. He, A. W. Cross, A. D. R. Phelps, C. G. Whyte, K. Ronald, and C. W. Robertson, "Experimental Study of Coaxial Free-Electron Maser Based on Two-Dimensional Distributed Feedback," Phys. Rev. Lett, vol. 96, no. 3, pp. 035002(1-4), Jan. 2006

[3] V. L. Bratman, G. G. Denisov, N. G. Kolganov, S. V. Mishakin, S. V. Samsonov, A. W. Cross, W. He, L. Zhang, M. McStravick, C. G. Whyte, A. R. Young, K. Ronald, C. W. Robertson, and A. D. R. Phelps, "Generation of $3 \mathrm{GW}$ Microwave Pulses in X-band from a Combination of a Relativistic Backward-Wave Oscillator and a Helical-Waveguide Compressor," Phys. of Plasmas, vol. 17, no. 11, pp. 110703 (1-3), Nov. 2010.

[4] J. Benford, H. Sze, W. Woo, R.R. Smith, and B. Harteneck, "Phase Locking of Relativistic Magnetrons," Phys. Rev. Lett., vol. 62, no.8, pp. 969-971, Feb. 1989

[5] H. Sze, D. Price, and B. Harteneck, "Phase Locking of 2 Strongly Coupled Vircators," J. Appl. Phys., vol. 67, no. 5, pp. 2278-2282, Mar. 1990.

[6] V. L. Bakunin, G. G. Denisov, and Yu.V. Novozhilova, "Frequency and Phase Stabilization of a Multimode Gyrotron with Megawatt Power by an External Signal," Tech. Phys. Lett., vol. 40, no. 5, pp. 382-385, May 2014.

[7] S. D. Korovin, A. A. Eltchaninov, V. V. Rostov, V. G. Shpak, M. I. Yalandin, N. S. Ginzburg, A. S. Sergeev, and I. V. Zotova, "Generation of Cherenkov Superradiance Pulses with a Peak Power Exceding the Power of the Driving Short Electron Beam," Phys. Rev. E, vol.74, no. 1, pp. 016501(1-8), Jul. 2006.

[8] V. V. Rostov, M. I. Yalandin, and G. A. Mesyats, "Repetitive Production of Nanosecond Gigawatt Microwave Pulses," IEEE Trans. Plasma Sci., vol. 36, no. 3, pp. 655-660, Jun. 2008 ,

[9] N. S. Ginzburg, S. P. Kuznetsov, and T. N. Fedoseeva, "Theory of Transit Processes in Relativistic BWT," Izv. Vyssh. Uchebn. Zaved., Radiofiz., vol. 21, no. 7, pp. 1037-1052, Jul. 1978.

[10] J. Jackson, Classical Electrodynamics (John Wiley \& Sons Inc., New York-London, 1962)

[11] N. S. Ginzburg, A. W. Cross, A. A. Golovanov, G. A. Mesyats, M. S. Pedos, A. D. R. Phelps, I. V. Romanchenko, V. V. Rostov, S. N. Rukin, K. A. Sharypov, V. G. Shpak, S. A. Shunailov, M. R. Ulmaskulov, M. I. Yalandin, and I. V. Zotova, "Generation of electromagnetic fields of extremely high intensity by coherent summation of Cherenkov superradiance pulses", Phys. Rev. Lett. 2015 (to be published 28 August 2015)

[12] G.V. Kisunko, Electrodynamics of hollow systems (VKAS, Leningrad, 1949).

[13] V. P. Tarakanov, User's Manual for Code KARAT (Berkeley Research Associates, Inc., Springfield, VA, USA, 1992).

[14] K. A. Sharypov, M. R. Ul'masculov, V. G. Shpak, S. A. Shunailov, M. I Yalandin, G. A. Mesyats, V. V. Rostov, and M. D. Kolomiets, "Current Waveform Reconstruction From an Explosively Emissive Cathode at a Subnanosecond Voltage Front" Rev. Sci. Instrum., vol. 85, no. 12, pp. 125104(1-6), Dec. 2014.

[15] M. I. Yalandin, A. G. Reutova, K. A. Sharypov, V. G. Shpak, S. A. Shunailov, M. R. Ul'maskulov, V. V. Rostov, and G. A. Mesyats, "Stability of Injection of a Subnanosecond High-Current Electron Beam and Dynamic Effects within its Rise Time," IEEE Trans. on Plasma Sci., vol. 38 , no. 10 , pp. 2559-2564, Oct. 2010.

[16] V. V. Rostov, A. A. El'chaninov, I. V. Romanchenko, and M. I. Yalandin, "A Coherent Two-Channel Source of Cherenkov Superradiance Pulses," Appl. Phys. Lett., vol. 100, no. 22, pp. 224102(14), May 2012.

[17] V. V. Rostov, A. A. Elchaninov, I. V. Romanchenko, S. A. Shunailov, M. R. Ul'maskulov, K. A. Sharypov, V. G. Shpak, S. N. Rukin, and M. I Yalandin, "Two-Channel Generator of the 8-mm Wavelength Range for
Radiation with Subgigawatt Power Level Pulses," Radiophysics and Quantum Electronics, vol. 56, no. 8-9, pp. 475-493, Jan. 2014.

[18] G. Sh. Boltachev, V. V. Rostov, K. A. Sharypov, S. A. Shunailov, V. G. Shpak, M. R. Ulmaskulov, and M. I. Yalandin, "Control of the Operation Mode of a Relativistic Ka-Band Backward-Wave Oscillator," IEEE Trans. on Plasma Sci., vol. 43, no. 8, pp. 2613 - 2620, Aug. 2015.

[19] V. V. Rostov, E. M. Totmeninov, and M. I. Yalandin, "High-Power Relativistic Microwave Sources Based on the Backward Wave Oscillator with a Modulating Resonant Reflector," Tech. Phys., vol. 53, no. 11, pp. 1471-1478, Nov. 2008

[20] W. Song, Y. Teng, Z. Q. Zhang, J. W. Li, J. Sun, C. H. Chen, and L. J. Zhang, "Rapid Startup in Relativistic Backward Wave Oscillator by Injecting External Backward Signal," Phys. of Plasmas, vol. 19, no. 8, pp. 083105 (1-4), Aug. 2012.

[21] E. B. Abubakirov, A. N. Denisenko, A. P. Konyushkov, E. I. Soluyanov, and V. V. Yastrebov, "Peculiarities of Operation of a Relativistic Backward-Wave Oscillator Driven by an External Electromagnetic Signal," Radiophysics and Quantum Electronics, vol. 57, no. 5, pp. 372378 , Oct. 2014

[22] M. I. Yalandin, S. A. Shunailov, M. R. Ul'maskulov, K. A. Sharypov, V. G. Shpak, V. V. Rostov, I. V. Romanchenko, A. A. El'chaninov, and A. I. Klimov, "Synphase Operation of Nanosecond Relativistic 37-GHz Backward-Wave Oscillators without Electrodynamic Coupling," Tech. Phys. Lett., vol. 38, no. 10, pp. 917-920, Oct. 2012.

[23] V. G. Shpak, S. A. Shunailov, M. I. Yalandin, and A. N. Dyadkov, "The RADAN SEF-303A, a Small High-Current Pulsed-Power Supply," Instrum. Exp. Tech., vol. 36, no. 1, pp. 106-111, Jan.-Feb.1993.

[24] S. N. Rukin, "High-Power Nanosecond Pulse Generators Based on Semiconductor Opening Switches (Review)," Instrum. Exp. Tech., vol. 42, no. 4, pp. 439-467, Jul.-Aug. 1999.

[25] I. V. Romanchenko, V. V. Rostov, V. P. Gubanov, A. S. Stepchenko, A.V. Gunin, and I. K. Kurkan, "Repetitive Sub-Gigawatt rf Source Based on Gyromagnetic Nonlinear Transmission Line," Review of Sci. Instrum., vol. 83, no.7, pp. 074705 (1-6), Jul. 2012.

[26] M. I. Yalandin, A. G. Reutova, M. R. Ul'maskulov, K. A. Sharypov, V. G. Shpak, S. A. Shunailov, A. I. Klimov, V. V. Rostov, and G. A. Mesyats, "Picosecond Stability of Injection of Parallel High Current Pulsed Electron Beams," Tech. Phys. Lett., vol. 35, no. 9, pp. 804-807, Sep. 2009.

[27] M. I. Yalandin, G. A. Mesyats, V. V. Rostov, K. A. Sharypov, V. G. Shpak, S. A. Shunailov, and M. R. Ulmasculov, "Suppression of Shunting Current in a Magnetically Insulated Coaxial Vacuum Diode," Appl. Phys. Lett., vol. 106, no. 23, pp. 233504(1-4), Jun. 2015.

[28] K. A. Sharypov, A. A. El'chaninov, G. A. Mesyats, M. S. Pedos, I. V. Romancheko, V. V. Rostov, S. N. Rukin, V. G. Shpak, S. A. Shunailov, M. R. Ul'masculov, and M. I. Yalandin, "Coherent Summation of KaBand Microwave Beams Produced by Sub-Gigawatt Superradiance Backward Wave Oscillators," Appl. Phys. Lett., vol. 103, no. 13, pp. 134103(1-4), Sep. 2013.

[29] N. S. Ginzburg, N. Yu. Novozhilova, I. V. Zotova, A. S. Sergeev, N. Yu. Peskov, A. D. R. Phelps, S. M. Wiggins, A. W. Cross, K. Ronald, W. He, V. G. Shpak, M. I. Yalandin, S. A. Shunailov, M. R. Ulmaskulov, and V. P. Tarakanov, "Generation of Powerful Subnanosecond Microwave Pulses by Intense Electron Bunches Moving in a Periodic Backward Wave Structure in the Superradiative Regime," Phys. Rev. E, vol. 60, no. 3, pp. 3297-3304, Sep. 1999.

[30] M. I. Yalandin, A. G. Reutova, K. A. Sharypov, V. G. Shpak, S. A. Shunailov, M. R. Ul'masculov, and G. A. Mesyats, "Microwave Breakdown of Air by Nanosecond and Subnanosecond Ka-Band Pulses," IEEE Trans. on Plasma Sci., vol. 38, no. 6, pp. 1398-1402, June 2010. 


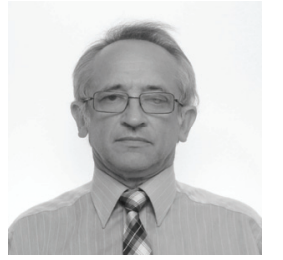

Naum S. Ginzburg was born in 1952. He received the M.Sc. degree in physics from Gorky State University in 1974, and the Ph.D. and Doctor of Science degrees from the Institute of Applied Physics, Russian Academy of Science, Nizhny Novgorod, in 1983 and 1994, respectively. He is a professor from 2002

At the Institute of Applied Physics he is now the Head of Department of Theory of Relativistic Microwave devices. His current research interests are high-power microwave electronics, including free electron laser, cyclotron resonance masers, gyrotrons, scattering electromagnetic waves by relativistic electron beams, as well as optical guiding and superradiance.

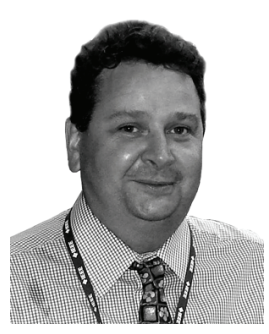

Adrian W. Cross received the B.Sc. (Hons.) degree in physics and the Ph.D. degree from the University of Strathclyde, Glasgow, U.K., in 1989 and 1993, respectively. He joined the Atoms, Beams, and Plasmas Group, University of Strathclyde, in 1993, initially as a Research Fellow and then a Lecturer, Senior Lecturer, and Reader with the Department of Physics in 2000, 2005, and 2007. From 2002 to 2007, he was an Engineering and Physical Science Advanced Fellow. He has been involved in various aspects of research on gyrotrons, cyclotron autoresonance masers, free-electron lasers, superradiant sources, gyrotron traveling wave amplifiers, and plasma applications. His current research interests include terahertz radiation sources and pseudospark physics.

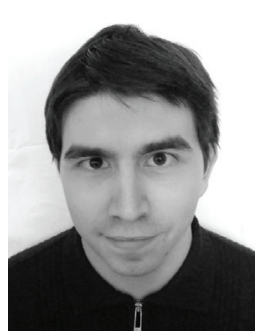

Anton A. Golovanov was born in 1992. He received the M.Sc. degree in physics from Lobachevsky State University of Nizhni Novgorod in 2015.

He currently works at the Institute of Applied Physics as a junior researcher. His fields of interest include relativistic electronics, superradiance of classical electron bunches and laser wakefield accelerators.

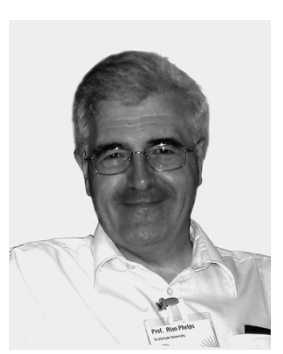

Alan D. R. Phelps (M'07) received the B.A degree (Hons.) in physics and the M.A. degree from Cambridge University, Cambridge, U.K., in 1966 and 1970, respectively, and the D.Phil. degree in plasma research from Oxford University, Oxford, U.K., in 1970.

He has been with the University of Strathclyde, Glasgow, U.K., since 1978, becoming a full Professor in 1993. His current research interests include high-power free-electron radiation sources and plasmas. Dr. Phelps is a fellow of the American Physical Society, the UK Institute of Physics, and the Royal Society of Edinburgh.

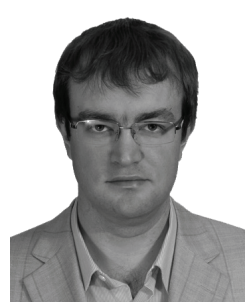

Ilya V. Romanchenko was born in 1983. He received the M.S. degree in physics from Tomsk State University in 2006 and the Candidate Sci. degree $(\mathrm{PhD})$ in physical electronics from the Institute of High Current Electronics, Russian Academy of Sciences, Tomsk in 2012. Since 2005, he has been with the Institute of High Current Electronics, where he is currently Junior Research Fellow at the Physical Electronics Department. His research interests include magnetic nonlinear transmission lines and high power microwaves.

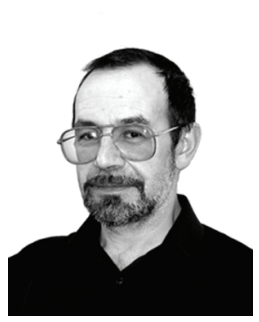

Vladislav V. Rostov received his M. Sc. degree in Plasma Physics from Novosibirsk State University in 1978, Cand. Sc. degree and Dr. Sc. degree from the Institute of High Current Electronics, Russian Academy of Sciences, Tomsk, in 1985 and 2001, respectively.

He has been with the Institute of High Current Electronics since 1978. He is heading a research team dealing with theoretical investigations in highpower microwave electronics.

Dr. Rostov was awarded Lenin Komsomol Prize in science in 1987 and State Prize of the Russian Federation in science in 2003.

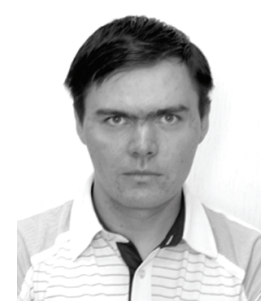

Konstantin A. Sharypov was born in 1977. He received his M. Sc. degree in Radiophysics from Moscow State University, Moscow, Russia, in 2000 and Cand. Sc. degree from the Institute of Electrophysics, Russian Academy of Sciences, Ekaterinburg, in 2013.

$\mathrm{He}$ is currently with the Institute of Electrophysics, Russian Academy of Sciences, where he is engaged in the development and maintenance of nanosecond and subnanosecond probes of high-voltage and RF pulses and of control systems for high-current electron accelerators.

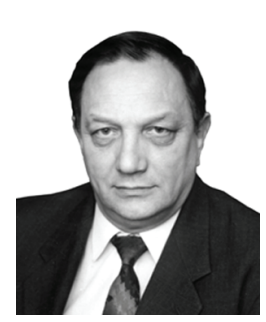

Valery G. Shpak was born in 1944. He received his M. Sc. and Cand. Sci. degrees in Electrovacuum Devices from the Institute of Radioelectronics, Tomsk, Russia, in 1967 and 1980, respectively, and Dr. Sc. degree from the Institute of High Current Electronics, Russian Academy of Science, Tomsk, in 1989.

He was formerly with the Institute of High Current Electronics in Tomsk, and since 1986, he has been Head of the Laboratory of Electron Accelerators at the Institute of Electrophysics, Russian Academy of Sciences, in Ekaterinburg. He is Director of the Institute of Electrophysics since 2004. Most of his publications are devoted to fast processes and compact pulsed power equipment operating on the nanosecond and subnanosecond scales.

Dr. Shpak was awarded State Prize of the Russian Federation in science in 1998 and Yablochkov Prize of the RAS in 2012. Since 1997, he has been Corresponding Member of the Russian Academy of Sciences.

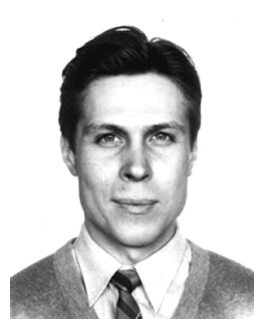

Sergei A. Shunailov was born in 1965. He received his M. Sc. degree in Physics from the Ural Polytechnic Institute in 1987 and Cand. Sc. degree from the Institute of Electrophysics, Russian Academy of Sciences, Ekaterinburg, in 1999.

At the Institute of Electrophysics, he is working in a team engaged in studying and developing compact high-voltage nanosecond and subnanosecond generators.

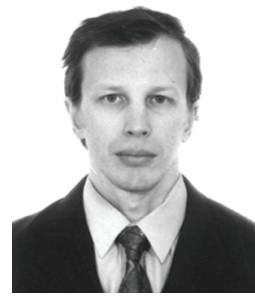

Marat R. Ulmaskulov was born in 1965 . He received his M. Sc. degree in Radio Engineering from Moscow State Technical University, Moscow, Russia, in 1992 and Cand. Sci. degree from the Institute of Electrophysics, Russian Academy of Sciences, Ekaterinburg, in 2002.

At the Institute of Electrophysics, he is working in a team engaged in studying and developing compact high-voltage nanosecond and subnanosecond devices, high-voltage and HPM diagnostic technique, and RF antenna systems. 


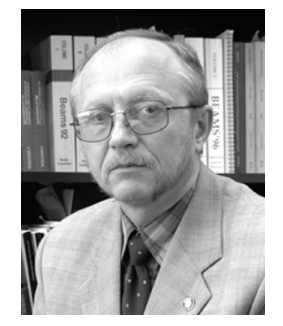

Michael I. Yalandin was born in 1956. He received his M. Sc. degree in Plasma Physics from

Novosibirsk State University in 1979 and his Cand. Sc. degree and Dr. Sc. degree from the Institute of High Current Electronics, Russian Academy of Sciences, Tomsk, in 1985 and 1996, respectively.

At the Institute of Electrophysics, Russian Academy of Sciences, he is heading a team engaged in studying and developing compact high-voltage nanosecond and subnanosecond modulators, pulsed electron accelerators, and high-power sources of coherent and broadband microwaves.

Dr. Yalandin was awarded Lenin Komsomol Prize in science in 1987, State Prize of the Russian Federation in science in 1998, Mikheev Prize of the Ural Branch of the RAS and Yablochkov Prize of the RAS, both in 2012. Since 2003, he has been Corresponding Member of the Russian Academy of Sciences.

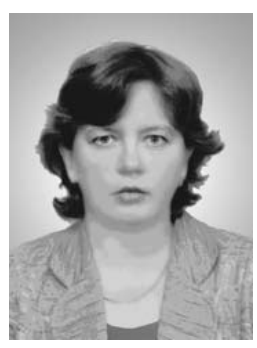

Irina V. Zotova was born in 1968. She received the M.Sc. degree in physics from Gorky State University in 1990, and the Ph.D. and Doctor of Science degrees from the Institute of Applied Physics, Russian Academy of Science, Nizhny Novgorod, in 1999 and 2015, respectively. At the Institute of Applied Physics she is now the leading research scientist. Her field of interests includes gyrotrons, relativistic electronics, optical guiding, superradiance of classical electron bunches. 